\title{
SLNB in melanoma-DFS a true and cost-effective benefit?
}

Alexander C. J. van Akkooi and Alexander M. M. Eggermont

With great interest have we noticed the correspondence of Faries et al. (MSLT-I - response of clinical trial investigators. Nat. Rev. Clin. Oncol. doi:10.1038/ nrclinonc.2014.65-c1), ${ }^{1}$ which relates their answers to previous criticism of the final analysis of their Multicenter Selective Lymphadenectomy Trial (MSLT-I) raised in our News \& Views article (van Akkooi, A. C. J. \& Eggermont, A. M. M. MSLT-1SNB is a biomarker, not a therapeutic intervention. Nat. Rev. Clin. Oncol. 11, 248-249; 2014)..$^{2}$ Importantly, a number of claims stated by these authors require a rebuttal.

First of all, the authors state that a reduction in recurrences induced by SLNB is not in question. Indeed, the FDA has approved disease-free survival (DFS) as a potentially valid end point for medical studies. However, it makes more sense that such an end point is used for a systemic adjuvant treatment rather than in a surgical trial, in which one group has undergone definitive surgery and the other group has not and, therefore, remains at risk. In 2007, the National Cancer Institute indicated that the nodal recurrences should be excluded from the DFS analyses of the MSLT-I data, because of the clear bias. ${ }^{3}$ Yet, the DFS benefit, which is presented in the final analyses of the MSLT-I data, is solely confined to the nodal recurrence rate.

In support of this claim, the appendix (Table S1a) of the New England Journal of Medicine paper on the final MSLT-I analysis by Morton et al. ${ }^{4}$ clearly reports the different relapse rates for the group that this intervention is aimed at (that is, patients with intermediate thickness, $1.2-3.5 \mathrm{~mm}$, melanoma). Local and/or in-transit recurrences were seen in $7.0 \%(n=54)$ versus $6.4 \%(n=32)$, nodal relapses $4.9 \%(n=38)$ versus $14.6 \%(n=73)$ and distant metastases rates were $13.9 \%(n=107)$ versus $11.2 \%(n=56)$ in the sentinel-lymph-node biopsy (SLNB) cohort compared with the observation group. ${ }^{4}$
No recurrences were seen in $74.2 \%$ $(n=571)$ versus $67.8 \%(n=339)$ of patients with sentinel-node-positive disease compared with observation group. ${ }^{4} P$-values were not given, but logical reasoning dictates that, either there were no statistically significant differences between the SLNB and observation arms with respect to local (and/or in-transit) and distant recurrences, or if anything, there was a detrimental effect of SLNB on outcome. The possible detrimental outcomes do not seem logical. Thus, the statistically significant $6.4 \%$ difference in recurrence rate and/or DFS can only be explained by the difference in the nodal recurrence rate, because in the SLNB group, the lymph-node metastases were already removed up-front, whereas in the observation group, the patients remain at risk of nodal recurrence. DFS is, therefore, not significantly improved by SLNB.

Secondly, the discrepancies with the amount of node-positive patients within the SLNB cohort versus the observation group and a claimed survival benefit for the node-positive SLNB subgroup remain unsolved. The authors repeat their claim that the difference in nodal-involvement rates has diminished with long-term follow-up and will eventually disappear. However, no data exist to show that, after 10 years, a false positive rate of $15.6 \%$ remains. ${ }^{2,5}$ Previous data showed a slowing down of relapse rates with increased follow-up time, ${ }^{6}$ and although new events might be seen in both groups, it is not expected that the false positive rate will ever reach $0 \%$. There are clearly concerns with the additional 'sophisticated' accelerated failure time latent subgroup analysis; $, 5,8$ this method has never been proven elsewhere, outside of the trial that produced the data on which the method was developed (in this case, the MSLT-I interim data from 2006).

Thirdly, the authors state that surgery or long-term follow-up are the only ways to stage disease. They ignore the less or noninvasive alternative staging methods, such as ultrasound-fine-needle aspiration cytology. An update by Voit et al. ${ }^{9}$ reported a sensitivity rate of $51 \%$ in 1,000 consecutive patients with stage I-II melanoma, before sentinelnode staging. The other $49 \%$ of patients with typically a smaller sentinel-node tumour burden can be simply followed up by repeat ultrasound assessments. ${ }^{10,11}$ This strategy has the potential to save $>50 \%$ of costs compared with surgical sentinel-node staging. ${ }^{6}$

Department of Surgical Oncology, Netherlands Cancer Institute, Antoni van Leeuwenhoek, Plesmanlaan 121, 1066 CX, Amsterdam, The Netherlands (A.C.J.v.A.). Cancer Institute, Gustave Roussy Cancer Campus, Grand Paris, 114 rue Édouard Vaillant, 94805 Villejuif, France (A.M.M.E.).

Correspondence to: A.C.J.v.A. a.v.akkooi@nki.nl

Competing interests

The authors declare no competing interests.

1. Faries, M. B. Cochran, A. J. \& Thompson, J. F. MSLT-I-response of clinical trial investigators. Nat. Rev. Clin. Oncol. http://dx.doi.org/ 10.1038/nrclinonc.2014.65-c1.

2. van Akkooi, A. C. J. \& Eggermont, A. M. M. Melanoma: MSLT-1-SNB is a biomarker, not a therapeutic intervention. Nat. Rev. Clin. Oncol. 11, 248-249 (2014).

3. Thomas, J. M. Concerns relating to the conduct and statistical analysis of the Multicenter Selective Lymphadenectomy Trial (MSLT-1) in patients with melanoma. J. Plast. Reconstr. Aesthet. Surg. 62, 442-446 (2009).

4. Morton, D. L. et al. Final trial report of sentinel-node biopsy versus nodal observation in melanoma. N. Engl. J. Med. 370, 599-609 (2014).

5. van Akkooi, A. C. Sentinel node followed by completion lymph node dissection versus nodal observation: staging or therapeutic? Controversy continues despite final results of MSLT-1. Melanoma Res. 24, 291-294 (2014).

6. van Akkooi, A. C., Voit, C. A., Verhoef, C. \& Eggermont, A. M. Potential cost-effectiveness of US-guided FNAC in melanoma patients as a primary procedure and in follow-up. Ann. Surg. Oncol. 17, 660-662 (2010).

7. Altstein, L. L. \& Li, G. Latent subgroup analysis of a randomized clinical trial through a semiparametric accelerated failure time mixture model. Biometrics 69, 52-61 (2013). 


\section{CORRESPONDENCE}

8. Altstein, L. L., Li, G. \& Elashoff, R. M. A method to estimate treatment efficacy among latent subgroups of a randomized clinical trial. Stat. Med. 30, 709-717 (2011).

9. Voit, C. A. et al. Ultrasound-guided fine needle aspiration cytology as an addendum to sentinel lymph node biopsy can perfect the staging strategy in melanoma patients. Eur. J. Cancer 50, 2280-2288 (2014).

10. Voit, C. A. et al. Ultrasound morphology criteria predict metastatic disease of the sentinel nodes in patients with melanoma. J. Clin. Oncol. 28, 847-852 (2010).
11. Voit, C. A. et al. Rotterdam Criteria for sentinel node (SN) tumor burden and the accuracy of ultrasound (US)-guided fine-needle aspiration cytology (FNAC): can US-guided FNAC replace SN staging in patients with melanoma? J. Clin. Oncol. 27, 4994-5000 (2009). 